JJ Igartua (2013): "Impacto actitudinal y canalización cognitiva de estereotipos sobre la inmigración a través de las noticias", en Revista Latina de Comunicación Social.

\title{
Impacto actitudinal y canalización cognitiva de estereotipos sobre la inmigración a través de las noticias
}

\author{
Attitudinal impact and cognitive channeling \\ of immigration stereotypes through the news
}

JJ Igartua [CV] Universidad de Salamanca - jigartua@usal.es

\begin{abstract}
s
[ES] Introducción. Tomando como base la investigación sobre el efecto framing y la investigación sobre el tratamiento de la inmigración, se analizan los procesos de recepción e impacto de las noticias sobre actos delictivos. Metodología. Se realizaron dos investigaciones experimentales en donde los participantes fueron expuestos a una noticia y posteriormente cumplimentaron un cuestionario con escalas de auto-informe. Se manipularon la mención del origen nacional/geográfico de los protagonistas de la noticia y la implicación con el tema de la noticia. Resultados. Se observa que cuando la implicación con el tema es baja, la presencia de información sobre el origen de los protagonistas de la noticia ejerce un efecto indirecto a través de impactar las actitudes. En cambio, cuando la implicación es alta el efecto indirecto se explica por la activación de corrientes de pensamiento que influyen en la formación y/o refuerzo de creencias negativas sobre la inmigración. Conclusiones. Los resultados son congruentes con un modelo dual de influencia de los encuadres noticiosos. Se constata con argumentos empíricos que la recomendación de no informar sobre el origen de los protagonistas en noticias sobre actos delictivos está justificada.

[EN] Introduction. Based on research on framing effects and on the news treatment of immigration, this article analyses the processes of reception and impact of news about crime. Method. We conducted two experimental studies in which participants were exposed to a news story and subsequently asked to answer a questionnaire with self-report scales. Two independent variables were manipulated in the news story: the reference to the protagonists' nationality and the place where the event took place (to influence readers' involvement with the topic). Results. The study indicates that when the involvement with the news story was low, the presence of information about the protagonists' nationality exerted an indirect effect through attitudinal impact. However, when involvement was high, the indirect effect was explained by the activation of trains of thought which influenced the formation and/or reinforcement of negative beliefs about immigration. Conclusions. The results are consistent with a dual model of news framing effects and empirically reinforce the recommendation of not revealing the nationality of the protagonists of a criminal act in a news story.
\end{abstract}




\section{Keywords}

[ES] Efecto de los encuadres noticiosos, clave grupal, implicación, inmigración, investigación experimental, análisis mediacional.

[EN] Framing effect, group cue, involvement, immigration, experimental research, mediational analysis.

\section{Contents}

[ES] 1. Introducción.1.1. Mecanismos explicativos del efecto framing. 1.2. Objetivos e hipótesis. 2. Estudio 1. 2.1. Método. 2.2. Resultados. 2.3. Conclusiones. 3. Estudio 2. 3.1. Método. 3.2. Resultados. 3.3. Conclusiones. 4. Conclusiones y discusión general. 5. Referencias bibliográficas [EN] 1. Introduction. 1.1. Mechanisms of framing effect. 1.2. Objectives e hypotheses. 2. Study 1. 2.1. Method. 2.2. Results. 2.3. Conclusions. 3. Study 2. 3.1. Method. 3.2. Results. 3.3. Conclusions. 4. Conclusions and general discussion. 5. References.

\section{Introducción}

El Consejo Audiovisual de Cataluña fue quizá el primer organismo que se pronunció en España sobre cómo debía informarse en los medios de comunicación sobre la inmigración. Así, en las recomendaciones específicas dirigidas a los profesionales de la información audiovisual se introducían dos que son relevantes en el contexto de los estudios que aquí se presentan: 1) la enfatización de las referencias a cuestiones como el origen o el color de la piel de los protagonistas de algunas noticias a menudo no añade información relevante al relato, no es estrictamente necesaria para la comprensión del hecho y, en cambio, actúa como refuerzo de prejuicios y como clave implícita de interpretación de intención negativa o condenatoria, y, 2) de manera parecida, y sin tener que renunciar sistemáticamente a las denominaciones colectivas, no es admisible la identificación de una minoría étnica o de un colectivo concreto de personas inmigradas con una determinada actividad ilegal, que puede acabar siendo considerada por la audiencia casi como un atributo o una característica del grupo en cuestión ${ }^{1}$ [1]. También desde OBERAXE (Observatorio Español del Racismo y la Xenofobia) se ha señalado que ha de evitarse la relación entre inmigración y delincuencia y no asociar la inmigración con lo negativo (Sendín e Izquierdo, 2008).

Los dos estudios experimentales que aquí se presentan ponen al descubierto los mecanismos (procesos mediadores de tipo actitudinal y cognitivo) que explican el impacto de la mención de la nacionalidad $\mathrm{u}$ origen de los protagonistas de una noticia sobre un acto delictivo en las creencias estereotípicas sobre la inmigración, tomando como marco de referencia la investigación sobre el efecto framing y el papel de las claves periféricas en el procesamiento de la información periodística. Los resultados de ambos estudios permiten avanzar en un modelo teórico sobre los efectos sociocognitivos de los encuadres noticiosos (modelo dual sobre el efecto de los encuadres noticiosos) y, además, demuestran con argumentos empíricos que la recomendación de no informar sobre el origen de los protagonistas de la noticia está justificada.

\subsection{Mecanismos explicativos del efecto framing}

Tankard (2001) afirma que encuadrar una información noticiosa implica: a) enfocar un tema de una cierta manera o perspectiva; b) fijar una agenda de atributos; c) elegir (seleccionar) ciertas palabras clave para confeccionar un discurso; d) manipular la saliencia de ciertos elementos o rasgos (dotar de énfasis a algunos de ellos); y, e) elaborar una idea organizadora central. Por otro lado, Entman (1993) considera que encuadrar "es seleccionar algunos aspectos de la realidad percibida y hacerlos más prominentes en un texto comunicativo, de manera que promuevan definiciones particulares de 
los problemas, interpretaciones causales, evaluaciones morales y/o recomendaciones para el tratamiento del asunto descrito" (p. 52). Por otro lado, algunos autores han señalado que el estudio de los encuadres noticiosos (y sus efectos), enlaza directamente con el denominado segundo nivel de la teoría de la Agenda Setting (McCombs, López-Escobar y Llamas, 2000; McCombs y Reynolds, 2002). Desde esta perspectiva, los encuadres noticiosos manipulan la saliencia de ciertos elementos o atributos con respecto a un tema o un acontecimiento (McCombs y Reynolds, 2002). En definitiva, encuadrar una información en un relato informativo está relacionado con dos operaciones básicas: seleccionar y enfatizar, palabras, expresiones e imágenes, para conferir un punto de vista, una perspectiva o un ángulo determinado a una información (De Vreese, 2003).

Los encuadres noticiosos pueden jugar diferentes roles, en particular, actuando como variables dependientes o como variables independientes (De Vreese, 2003; Scheufele, 1999). En primer lugar, entendidos como variables dependientes los encuadres están contenidos en las noticias y son el resultado de los procesos de producción de las mismas. Como variables independientes, los encuadres son concebidos como propiedades de los textos noticiosos que condicionan los procesos de recepción e impacto de las noticias. Esta línea de investigación enlaza con el análisis del denominado efecto framing (Iyengar, 1991; De Vreese, 2003; Price y Tewksbury, 1997; Scheufele, 2000). Scheufele (2000) señala que los encuadres noticiosos pueden influir en el contenido del pensamiento de las personas (en sus respuestas cognitivas) y, por tanto, canalizar la forma de reflexionar de las personas sobre los asuntos tratados en una noticia; también pueden ejercer un efecto considerable en cómo pensar el mundo social y producir un cambio o un impacto en las opiniones y actitudes debido a sutiles alteraciones en la definición de un problema en una noticia. Finalmente, también se ha establecido que los encuadres noticiosos pueden influir en las respuestas o reacciones emocionales, es decir, que en función de cómo se enfoque un asunto en una noticia se inducirán diferentes emociones hacia el objeto tratado en la misma (Gross y Brewer, 2007; Gross y D’Ambrosio, 2004; Igartua, Moral y Fernández, 2011).

El debate actual en este campo se centra en analizar los mecanismos explicativos del efecto de los encuadres noticiosos ${ }^{2}$ [2] (Igartua y Cheng, 2009; Price y Tewksbury, 1997; Scheufele, 2000). Matthes (2007) indica que la mayor parte de la teorización sobre el efecto framing se ha desarrollado a partir del concepto de accesibilidad (memory-based model). Sin embargo, también se concibe que el efecto framing se vincula con la aplicabilidad del conocimiento que se activa durante la recepción de una noticia, es decir, con la activación de "corrientes de pensamiento" que influyen de manera espontánea en la formación de actitudes y creencias, gracias al énfasis de ciertos atributos en la noticia (on-line model) (Price y Tewksbury, 1997; Price, Tewksbury y Powers, 1997). Una tercer postura es considerar que el efecto framing se vincula con los procesos de persuasión y, en este contexto, se ha planteado que puede estar gobernado por el procesamiento heurístico (Igartua y Cheng, 2009; Igartua, Moral y Fernández, 2011; Perse, 2001; Von Sikorski y Schierl, 2012). Esto significa que el impacto de los encuadres estaría relacionado con la puesta en marcha de procesos cognitivos que no implican un análisis exhaustivo de la información, es decir, con la activación de atajos mentales y procesos de modelado actitudinal, de modo que la actitud implícita en la noticia es asimilada de manera automática, sin que se produzca un proceso de reflexión o elaboración, por parte los individuos. Así, se considera que cuando las personas no están especialmente motivadas para procesar la información (que podría ser la posición por defecto, dados los bajos índices de retención de la información; Machill, Köhler y Waldhauser, 2007), la presencia de claves periféricas (peripheral cues) en el mensaje noticioso puede condicionar los efectos socio-cognitivos.

Perse (2001) ha indicado que el efecto framing podría explicarse a partir del Modelo de Probabilidad de Elaboración (Elaboration Likelihood Model, ELM) de Petty y Cacioppo (1986), siendo el resultado de un procesamiento vía ruta periférica. El ELM plantea dos diferentes rutas responsables del cambio de actitudes: una ruta de procesamiento central y una ruta de procesamiento periférico. 
La ruta central implica que el receptor del mensaje intenta realizar una evaluación crítica y exhaustiva del mismo. Este tipo de procesamiento sistemático tiene lugar cuando las personas se enfrentan a contenidos que suscitan en ellas un gran interés o con los que mantienen una alta implicación porque son considerados temas importantes. El principal mecanismo del cambio de actitud en este contexto es la cantidad y tipo de respuestas cognitivas elaboradas por los individuos (qué y cuánto se reflexiona durante el procesamiento del mensaje). En cambio, el procesamiento vía ruta periférica es de naturaleza superficial y automática, se basa en claves periféricas (quién dice qué, cómo se dice, etc.) y se pone en marcha cuando los individuos se enfrentan a contenidos de baja implicación, es decir, se exponen a mensajes que abordan temas que no suscitan interés o se consideran poco relevantes desde un punto de vista personal. En este contexto, el procesamiento heurístico (que opera a través de la ruta periférica) se refiere a la aplicación de reglas simples de decisión sobre aspectos no centrales del mensaje (por ejemplo, "si la noticia aparece publicada en un diario de prestigio, frente a uno sensacionalista, es más probable que la información sea cierta y esté contrastada").

Una clave periférica en el contexto de las noticias sobre inmigración es la alusión al origen nacional o geográfico de los inmigrantes (group cue) (Brader, Valentino y Suhay, 2008). En este contexto, Igartua y Cheng (2009) observaron que la presentación incidental de información sobre el origen geográfico o nacional de inmigrantes con una peor imagen entre la opinión pública (marroquíes frente a latinoamericanos) en el contexto de una noticia sobre las consecuencias de la inmigración para el país, exacerbaba el prejuicio y reforzaba actitudes y creencias negativas. Estos resultados son convergentes con los estudios sobre el papel de las claves (textuales o visuales) sobre el origen étnico o grupal de los protagonistas de las noticias en la activación de los estereotipos (por ejemplo, Gilliam, Iyengar, Simon y Wright, 1996; Peffley, Shields y Williams, 2001). Se ha observado que la manipulación delgrupo étnico de un sospechoso (afroamericano versus blanco) en el contexto de una noticia sobre el crimen, influye en la preocupación por el crimen y en las atribuciones sobre las causas del mismo (Gilliam et al., 1996) y sesga la evaluación efectuada sobre dicho sospechoso (Peffley et al., 2001). Desde este marco cognitivo, se considera que la influencia cognitiva de las noticias se explica porque, en la interacción con los contenidos periodísticos, la mayor parte del tiempo se opera con baja motivación y capacidad de procesamiento. En esas condiciones, el procesamiento heurístico es el que acaba dominando (frente al procesamiento sistemático). Por tanto, un efecto principal del consumo de noticias consiste en facilitar la accesibilidad de ciertas informaciones y, con ello, estimular un procesamiento más superficial y menos profundo (mediante la aplicación de heurísticos) sobre el mundo social (Perse, 2001).

Ahora bien, ¿qué ocurre cuando la implicación con el tema de la noticia es alta y qué papel juegan las calves periféricas en dicho contexto? En primer lugar, cabe argumentar que si las noticias se procesaran de manera sistemática (vía ruta central) los efectos de claves periféricas (como la alusión al origen étnico, geográfico o nacional de los protagonistas de la información) serían poco relevantes, ya que lo verdaderamente importante en este contexto es la cantidad y tipo de reflexión que está determinado a su vez por la calidad de la información contenida en la notica. De este modo, cuando se produce un procesamiento sistemático de la información, el impacto de los encuadres noticiosos se explica por la mediación cognitiva; es decir, las respuestas cognitivas ejercerán un papel mediador. Sin embargo, estar altamente implicado con un tema no garantiza que se produzca un procesamiento objetivo de la información. Según el ELM, la implicación con el tema del mensaje determina no sólo el grado o intensidad de elaboración o reflexión (cuánto se reflexiona durante la exposición al mensaje), sino que también afecta a la dirección de la elaboración (Petty, Priester y Wegener, 1994).

Se ha señalado que el tipo de elaboración cognitiva puede ser de dos tipos: objetivo y sesgado. El procesamiento cognitivo objetivo implica que el individuo realizará un análisis concienzudo, crítico 
y teniendo en cuenta la evidencia presentada en el mensaje. Será un tipo de procesamiento guiado por los datos (bottom-up processing), ya que se asume que la elaboración es relativamente imparcial. En cambio, el procesamiento cognitivo sesgado se desencadenará ante circunstancias en las que la valoración de la evidencia presente en el mensaje no constituye un elemento central para el individuo. De este modo, la persona procesa la comunicación guiado casi exclusivamente por el esquema, la actitud o el conocimiento previo sobre el tema y pretende mantener la consistencia en su sistema de creencias y defender sus actitudes iniciales (top-down processing). Desde este punto de vista, se puede asumir que la presencia de una clave periférica en el contexto de una noticia sobre un acto delictivo (por ejemplo, indicando que los delincuentes eran inmigrantes o señalando la nacionalidad de los mismos) cumpliría la función de facilitar el procesamiento cognitivo sesgado, produciéndose un proceso de canalización cognitiva. De este modo, las claves periféricas pueden influir en la dirección de las respuestas cognitivas y éstas, a su vez, inducir un mayor impacto en las opiniones o creencias.

\subsection{Objetivos e hipótesis}

El objetivo principal de los dos estudios que se presentan fue contrastar el efecto diferencial de la inclusión de información sobre el origen de los delincuentes (group cue) en una noticia sobre un incidente delictivo en personas con alta y baja implicación con el acontecimiento relatado. Se asume que el efecto framing puede explicarse teniendo en cuenta los mismos mecanismos que explican el impacto actitudinal provocado por las comunicaciones persuasivas. De este modo, la presencia de claves periféricas se considera un elemento que, de forma rutinaria, se está utilizando para construir noticias que vinculan inmigración con delincuencia. Hasta el punto que se ha llegado a convertir en un atributo característico de este tipo de encuadre noticioso de la inmigración. En este contexto, cabe hipotetizar que el efecto de la mención del origen nacional de los delincuentes en una noticia sobre un incidente delictivo podría explicarse por diferentes mecanismos en función del grado de implicación de los individuos.

La implicación o relevancia personal se refiere a la importancia intrínseca que un determinado asunto a acontecimiento posee para una persona (Johson y Eagly, 1990). Si el individuo cree que la cuestión tratada en una noticia puede tener consecuencias importantes para su propia vida, se dirá que nos encontramos ante una mensaje de alta implicación. Por el contrario, si el tema o acontecimiento no afecta al individuo, dicho mensaje carecerá de relevancia. Dado que la implicación con un tema es una variable situacional es posible su manipulación experimental. En las dos investigaciones que se presentan, la implicación se ha manipulado variando el contexto en el que se producían los hechos narrados en la noticia: cercano o alta implicación (el incidente delictivo se produjo en la misma ciudad en la que vive el individuo) o lejano, de baja implicación (el incidente tuvo lugar en otra ciudad distante, perteneciente a otra comunidad autónoma). Este procedimiento de manipulación de la implicación mediante sutiles alteraciones en el texto es el procedimiento habitual utilizado en las investigaciones sobre persuasión (Igartua, Cheng y Lopes, 2003; Petty y Cacioppo, 1986).

De este modo, los individuos expuestos a una noticia de baja implicación "se dejarían llevar" por los aspectos periféricos de la noticia (la mención sobre el origen de los delincuentes), observándose un mayor impacto en actitudes, creencias y emociones hacia los inmigrantes. De este modo, se plantearon las siguientes hipótesis:

H1: En una noticia sobre un acto delictivo, la presencia de información sobre el origen nacional de los delincuentes (definidos como inmigrantes), estimulará una actitud más negativa hacia la inmigración únicamente cuando se induzca una baja implicación en los participantes (estudio 1).

H2: En una noticia sobre un acto delictivo, la presencia de información sobre el origen nacional de los delincuentes (definidos como inmigrantes), provocará un mayor grado de acuerdo con las 
creencias negativas y estereotípicas sobre las consecuencias de la inmigración únicamente cuando se induzca una baja implicación en los participantes (estudio 1).

H3: En una noticia sobre un acto delictivo, la presencia de información sobre el origen nacional de los delincuentes (definidos como inmigrantes), provocará una mayor vivencia de emociones negativas hacia los inmigrantes y una menor intensidad en las emociones positivas, únicamente cuando se induzca una baja implicación en los participantes (estudio 1).

Un segundo objetivo de ambos estudios fue analizar los mecanismos o procesos mediadores que explican el impacto de la mención del origen nacional de los protagonistas de la noticia en la formación y/o refuerzo de creencias estereotípicas sobre los inmigrantes. Cabía esperar que el efecto de la mención de información sobre el origen de los protagonistas de la noticia fuera diferente en personas de baja y alta implicación. En las personas con baja implicación, la mención expresa de información sobre el origen de los protagonistas de la noticia actuará como una clave periférica, de modo que activará una actitud negativa hacia la inmigración y esta actitud se asociará a su vez a un mayor grado de acuerdo con las creencias negativas y estereotípicas sobre la inmigración. En cambio, en las personas con alta implicación, la presencia de dicha información sesgará el procesamiento de la información, es decir, producirá un efecto a partir de provocar un proceso de canalización cognitiva lo que determinará a su vez un mayor acuerdo con las creencias estereotípicas sobre la inmigración. En este sentido se plantearon las siguientes hipótesis:

H4: El efecto de la mención del origen nacional de los protagonistas de la noticia sobre las creencias estereotípicas sobre la inmigración estará mediado por la actitud hacia la inmigración, pero únicamente cuando la implicación con el tema de la noticia sea baja (estudio 1).

H5: En una noticia sobre un acto delictivo, la presencia (versus ausencia) de información sobre el origen nacional de los delincuentes (definidos como inmigrantes), provocará un efecto de canalización cognitiva y respuestas cognitivas más críticas hacia la inmigración, únicamente entre los participantes a los que se induzca alta implicación con la noticia (estudio 2).

H6: El efecto de la mención del origen nacional de los protagonistas de la noticia sobre las creencias estereotípicas sobre la inmigración estará mediado por las respuestas cognitivas suscitadas por la lectura de la noticia, pero únicamente cuando la implicación con el tema de la noticia sea alta (estudio 2).

\section{Estudio 1}

\subsection{Método}

El estudio 1 consistió en una investigación experimental, en la que participaron 237 estudiantes de la Universidad Europea Miguel de Cervantes (de Valladolid ${ }^{3}$ [3]), siendo el $47.3 \%$ mujeres, y con una media de edad de 20.95 años ( $D T=2.52$; de 18 a 36 años).

Se utilizó un diseño 2 x 2, en función de dos manipulaciones experimentales realizadas en una noticia que aludía a un acontecimiento delictivo. La primera variable independiente se relacionaba con la manipulación del group cue, es decir, la mención de información en la noticia sobre el origen geográfico de los delincuentes que protagonizaban el incidente narrado, estableciéndose dos condiciones "no se indica el origen de los delincuentes" versus "se indica que los delincuentes son inmigrantes". La segunda variable independiente era la implicación con el tema de la noticia: baja (el suceso delictivo sucedía en Barcelona) o alta (el suceso delictivo sucedía en Valladolid, la ciudad de los participantes en el estudio). 
Las variables dependientes fueron evaluadas mediante un cuestionario split-ballot que fue aplicado en varias aulas de la Universidad Europea Miguel de Cervantes de Valladolid. En cada aula, los participantes fueron aleatorizados a las cuatro condiciones experimentales. En la portada del cuestionario se entregaba información sobre los objetivos del estudio. En la segunda página se incluía la noticia experimental que en las cuatro condiciones presentaba el mismo titular: Una mujer, en estado muy grave al ser brutalmente atacada por dos ladrones que asaltaron su domicilio. La noticia utilizada en el estudio se basaba en una noticia real que, con el mismo titular, había sido publicada en la versión impresa del diario El País el 18 de junio de $2006^{4}$ [4] (únicamente el 5.5\% de los participantes declaró que ya había leído con anterioridad la noticia). Una vez leída la noticia experimental, los participantes debían cumplimentar las preguntas del cuestionario relacionadas las variables dependientes, de chequeo de la manipulación y de control:

Emociones sentidas durante la lectura de la noticia (Igartua et al., 2003). Se utilizó una escala compuesta por 10 ítems, de modo que cada uno de ellos se refería a una emoción (interés, alegría, sorpresa, tristeza, cólera o ira, repugnancia o asco, desprecio o menosprecio, miedo o temor, vergüenza y culpa). Los participantes debían indicar en qué medida la lectura de la noticia les había hecho sentir dichas emociones (desde 1 "nada" hasta 5 "mucho").

Evaluación de la noticia. Se utilizó un diferencial semántico compuesto por 9 escalas bipolares (de 7 puntos) en cuyos extremos figuraban los adjetivos antónimos "clara-confusa", "de lectura fácil-de lectura difícil", "profunda-superficial", "objetiva-sesgada", "compleja-simple", "contextualizadadescontextualizada", "imprecisa-rigurosa", "atractiva-repulsiva" y "entretenida-aburrida".

Interés suscitado por la noticia. Se preguntó a los participantes "valora el grado de interés personal que te ha suscitado la noticia" (desde 0 "ningún interés" hasta 10 "mucho interés").

Actitud hacia la inmigración. Se preguntó: "como sabes, todos los países desarrollados reciben inmigrantes. ¿Crees que, en términos generales, la inmigración es más bien positiva o más bien negativa para España?” (desde 0 “muy negativa” hasta 10 “muy positiva”).

Creencias sobre las consecuencias de la inmigración. Se construyó una escala con 8 afirmaciones sobre las consecuencias de la inmigración en España (Cea D’Ancona, 2004; Igartua y Cheng, 2009). Se preguntaba por el grado de acuerdo o desacuerdo (1=total desacuerdo, $5=$ total acuerdo) con las afirmaciones: 1) "los inmigrantes desempeñan trabajos que los españoles no quieren", 2) "en España, hoy en día, se siguen necesitando trabajadores inmigrantes", 3) "el aumento de la inmigración favorece el incremento de la delincuencia", 4) "una sociedad sin inmigrantes es difícil que funcione y avance", 5) "existe una estrecha relación entre inmigración e inseguridad ciudadana", 6) "el aumento de los inmigrantes favorece la economía del país", 7) "cuando pienso en los inmigrantes enseguida me vienen a la memoria todos los problemas que ocasionan" y 8) "en general, los inmigrantes están contribuyendo al desarrollo del país". Un análisis factorial de componentes principales (rotación varimax) extrajo dos factores (69.94\% de la varianza). El primer factor (ítems 1, 2, 4, 6 y 8) aludía a la creencia "la inmigración supone una contribución económica necesaria para el país" $(\alpha=.85)$; el segundo (ítems 3,5 y 7) se refería a la creencia "la inmigración favorece el incremento de la delincuencia y la inseguridad ciudadana" $(\alpha=.79)$.

Emociones sentidas hacia los inmigrantes. Esta escala fue elaborada a partir de la escala de prejuicio sutil afectivo de Navas, García, Rojas, Pumares y Cuadrado (2006). Los participantes debían expresar en qué medida los inmigrantes les inspiraban las siguientes emociones: miedo, admiración, desconfianza, inseguridad, simpatía, incomodidad e indiferencia (desde 1 "nada" hasta 5 "mucho"). Un análisis factorial de componentes principales, con rotación varimax, extrajo dos factores que explicaban $66.97 \%$ de la varianza: a) emociones negativas de miedo, desconfianza, inseguridad, incomodidad e indiferencia $(\alpha=.81)$, y b) emociones positivas de admiración y simpatía $(\alpha=.76)$. 
Interés por el tema de la inmigración. Se preguntaba: “¿cuánto interés te suscita el tema de la inmigración?" (desde 1 "nada" hasta 5 "mucho"; $M=3.66, D T=0.85$ ).

Participación en conversaciones sobre el tema de la inmigración. A los participantes se les planteó la siguiente pregunta: "¿Alguna vez has participado activamente, dando tu opinión, en conversaciones o discusiones sobre el tema de la inmigración?" (desde 1 "nunca" hasta 5 "muchas veces"; $M=2.98, D T=1.26$ ).

Nivel de contacto con inmigrantes. Se preguntaba al sujeto si mantenía, o había mantenido en el pasado $(1=$ sí, $0=n o$ ), algún tipo de relación familiar, de amistad, en el trabajo, de estudios o de vecindad con inmigrantes (Cea D'Ancona, 2004). Se creó un índice de contacto personal con inmigrantes a partir de la suma simple de las cinco variables dicotómicas consideradas $(M=1.85$, $D T=1.28)$.

Las variables emociones sentidas durante la lectura de la noticia y el interés suscitado por la noticia se utilizaron en para contrastar la eficacia de la manipulación de experimental relacionada con la implicación con la noticia. La medición de la evaluación de la noticia se incluyó para comprobar la equivalencia de las cuatro versiones de la noticia. Las variables interés por el tema de la inmigración, participación en conversaciones sobre el tema de la inmigración, nivel de contacto con inmigrantes, junto con el sexo y la edad de los encuestados, se consideraron variables control y se incluyeron para evaluar la eficacia de la aleatorización de los participantes de las condiciones experimentales. Finalmente, las variables actitud hacia la inmigración, creencias sobre las consecuencias de la inmigración y emociones sentidas hacia los inmigrantes constituyeron las variables dependientes del estudio.

\subsection{Resultados}

En primer lugar, se realizó un análisis de varianza de un factor (ANOVA) en el que se incluyó como variable independiente la condición experimental ${ }^{5}$ [5] y como variables dependientes los nueve indicadores de evaluación de la noticia. Los resultados mostraron que no existían diferencias estadísticamente significativas entre las cuatro versiones de la noticia experimental en ninguna de las variables de evaluación de la noticia (en todos los análisis $p>.220$ ). Estos resultados indican que las cuatro noticias experimentales, a pesar de contener dos manipulaciones, no generaron diferencias en cuanto a su valoración general y por tanto se comportaron como estímulos homogéneos. Tampoco se observaron diferencias significativas entre los cuatro grupos experimentales en función del sexo, la edad, el interés por el tema de la inmigración, la participación en conversaciones sobre el tema de la inmigración y el nivel de contacto con inmigrantes (en todos los análisis $p>$.250). Por tanto, se puede concluir que el procedimiento de aleatorización de los participantes a las condiciones fue exitoso y condujo a la formación de cuatro grupos homogéneos en variables relevantes.

Con objeto de contrastar la eficacia de la manipulación de la implicación, se analizó el efecto de dicha variable en el interés suscitado por la noticia y en la intensidad de las emociones sentidas. No se observaron diferencias significativas en el interés ni en la intensidad de emociones suscitadas por la lectura de la noticia en función de la implicación (en todos los análisis $p>.120$ ). A raíz de estos resultados se puede concluir que la manipulación de la implicación con la noticia no produjo el efecto esperado en los participantes, de modo que tanto la noticia situada en Valladolid como la emplazada en Barcelona provocaron un mismo patrón de emociones y el mismo grado de interés. A pesar de ello, se procedió al contraste de las hipótesis.

Para contrastar las hipótesis 1 se efectuó un análisis factorial de la varianza para un diseño 2 x 2 de medidas independientes, siendo la variable dependiente la actitud hacia la inmigración. Se observó un efecto de interacción estadísticamente significativo entre las dos variables independientes $(F(1$, 
233) $=4.36, p<.038, \eta_{\mathrm{p}}{ }^{2}=.018$ ), lo que confirma la hipótesis 1 y pone de manifiesto que entre los participantes con baja implicación el efecto dela mención del origen geográfico de los delincuentes (que estos eran inmigrantes) sobre la actitud hacia la inmigración fue mayor que entre los sujetos con alta implicación (Figura 1). Sin embargo, con respecto a la creencia negativa "la inmigración favorece el incremento de la delincuencia y la inseguridad ciudadana" no se observó un efecto de interacción significativo entre ambas variables independientes, por lo que la hipótesis 2 no recibió apoyo empírico.

Figura 1.Efecto de la información sobre el origen de los delincuentes y de la implicación con el tema de la noticia en las actitudes hacia la inmigración

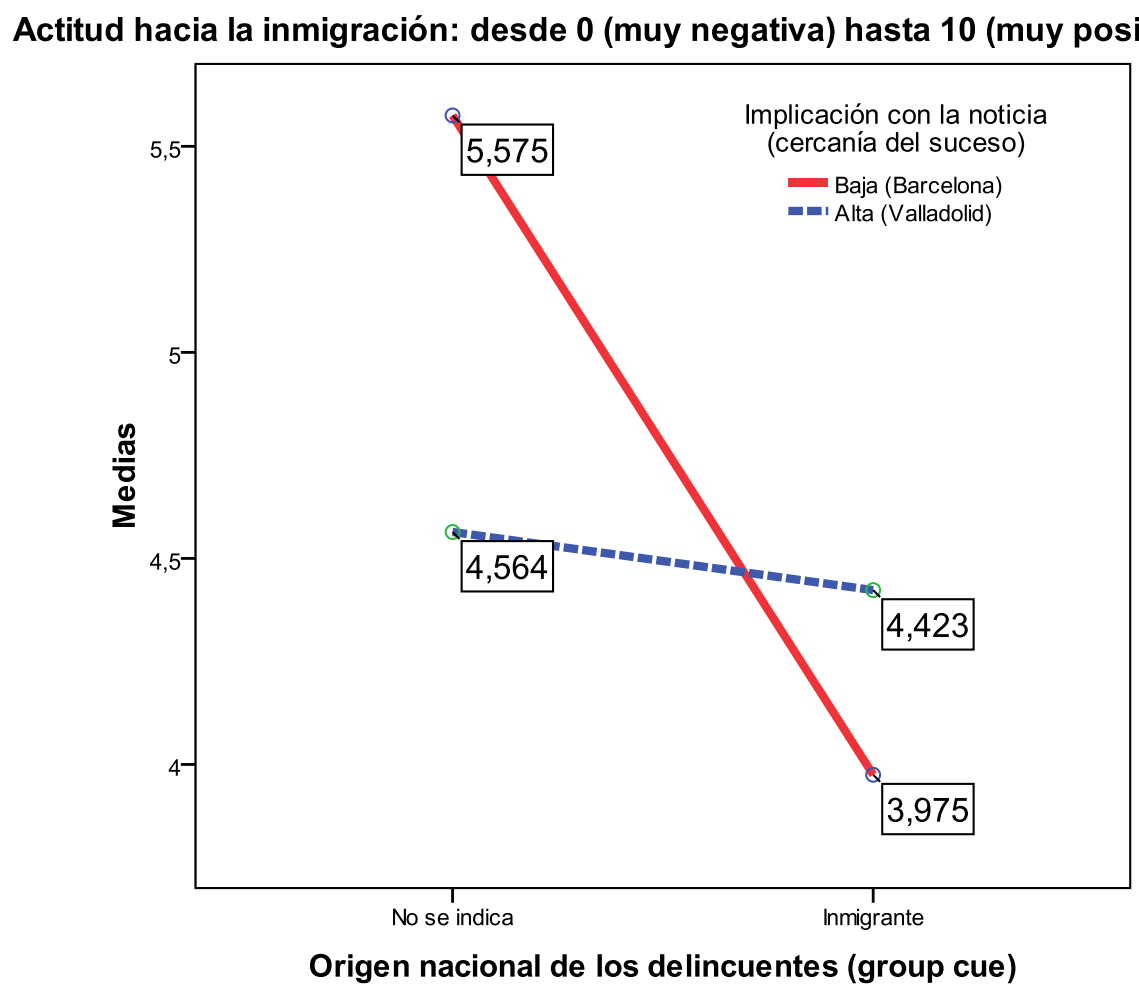

En cuanto al impacto en las emociones hacia los inmigrantes, se observó un efecto de interacción estadísticamente significativo entre las dos variables independientes $(F(1,230)=4.61, p<.033$, $\eta_{\mathrm{p}}{ }^{2}=.020$ ) en las emociones negativas hacia los inmigrantes (miedo, desconfianza, inseguridad, incomodidad e indiferencia). Los participantes con baja implicación informaron de más emociones negativas hacia los inmigrantes cuando leyeron una noticia que indicaba que los delincuentes eran extranjeros, frente a los participantes expuestos a una noticia que no indicaba nada al respecto (Figura 2). 
Figura 2. Efecto de la información sobre el origen de los delincuentes y de la implicación con el tema de la noticia en las emociones negativas hacia los inmigrantes

\section{Emociones negativas hacia los inmigrantes: miedo, desconfianza, inseguridad, incomodidad, indiferencia}

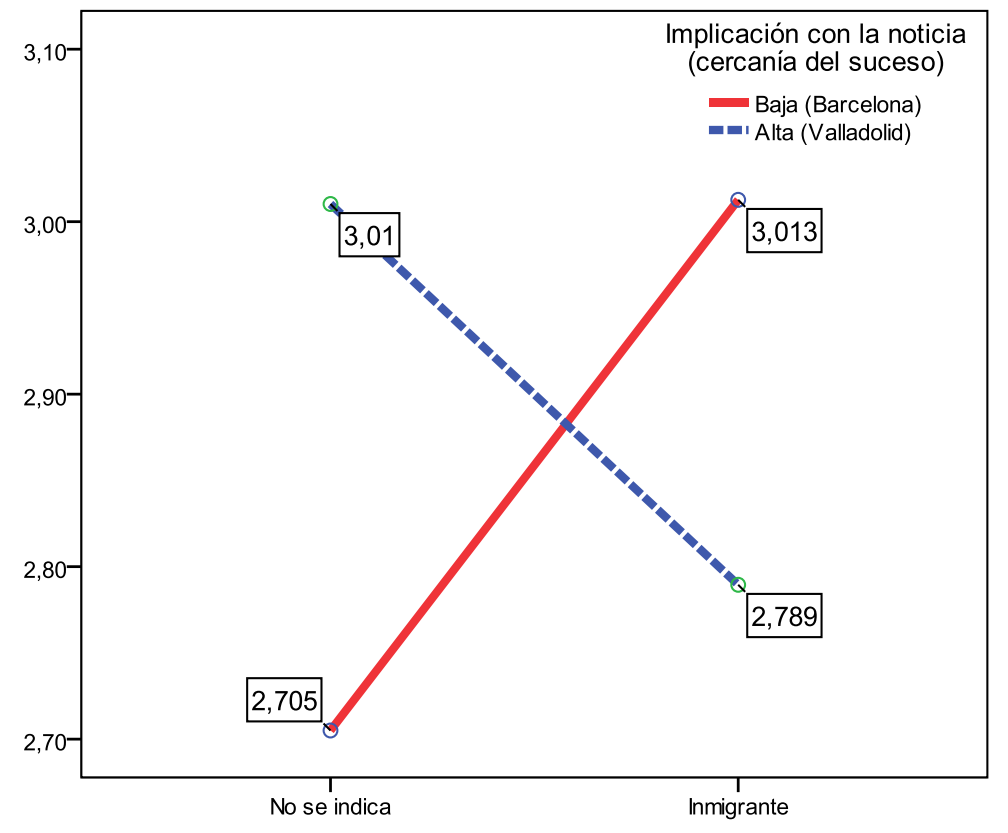

Origen nacional de los delincuentes (group cue)

Para contrastar la hipótesis 4 se utilizó la macro PROCESS (modelo 4, modelo mediacional simple) para SPSS elaborada por Hayes (2013) que permite estimar los efectos indirectos en modelos mediacionales, utilizando el método bootstrapping (Hayes, 2009). El modelo mediacional hipotetizado incluía las siguientes variables: la presencia o ausencia sobre de información sobre el origen nacional de los delincuentes (variable independiente, codificada como variable dummy, siendo $1=$ en la noticia se alude a que los delincuentes son inmigrantes, $0=$ no se menciona el origen nacional de los delincuentes), la actitud hacia la inmigración (variable mediadora) y el grado de acuerdo con la creencia negativa y estereotípica "la inmigración favorece el incremento de la delincuencia y la inseguridad ciudadana" (variable dependiente). El modelo mediacional propuesto fue computado de manera separada para los participantes de alta y baja implicación.

El análisis mediacional ejecutado entre los participantes de baja implicación reveló que la actitud hacia la inmigración mediaba la relación entre la presencia de información sobre el origen nacional de los protagonistas de la noticia y un mayor acuerdo con la creencia estereotípica negativa "la inmigración favorece el incremento de la delincuencia y la inseguridad ciudadana", ya que el efecto indirecto (calculado utilizando 10.000 bootstrap samples) resultó estadísticamente significativo $\left(B_{\text {efecto indirecto }}=.44, S E=.14,95 \% C I[.18, .74]\right)^{6}[6]$. De este modo, la mención en la noticia de que los delincuentes eran inmigrantes inducía una actitud más negativa hacia la inmigración $(B=-1.62$, $p<.001)$ y ésta, a su vez, se asociaba a un mayor acuerdo con que "la inmigración favorece el incremento de la delincuencia y la inseguridad ciudadana" $(B=-.27, p<.001)$ (véase Figura 3). Sin embargo, el análisis mediacional entre los participantes de alta implicación reveló que la actitud hacia la inmigración no mediaba la relación entre la presencia de información sobre el origen nacional de los protagonistas de la noticia y un mayor acuerdo con la creencia estereotípica negativa 
"la inmigración favorece el incremento de la delincuencia y la inseguridad ciudadana", ya que el efecto indirecto no fue estadísticamente significativo $\left(B_{\text {efecto indirecto }}=.03, S E=.09,95 \% C I[-.15, .22]\right.$. De este modo, la hipótesis 4 recibió apoyo empírico.

Figura 3. Análisis mediacional (baja implicación, $\mathrm{n}=119$, estudio 1): la actitud hacia la inmigración como variable mediadora en la relación entre la mención de la información sobre el origen nacional de los protagonistas de la noticia y las creencias estereotípicas negativas sobre la inmigración

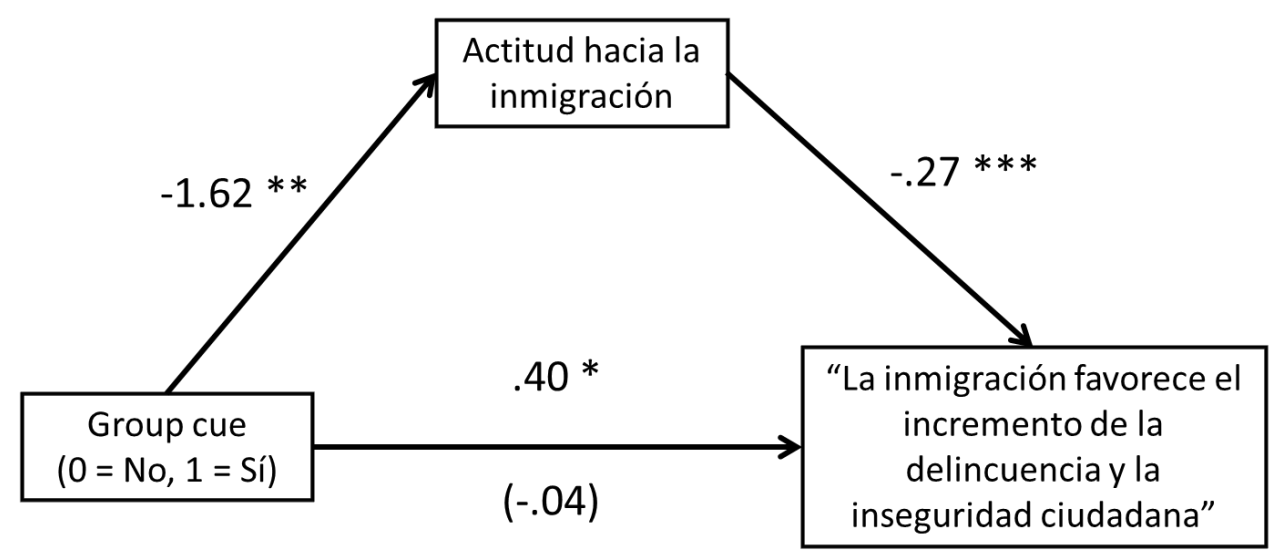

Group cue -> Actitud hacia la inmigración -> La inmigración favorece la delincuencia

$$
B \text { efecto indirecto }=.44, S E=.19,95 \% C I[.18, .74]
$$

$N o t a$. En la figura se muestran los coeficientes de regresión no estandarizados, $B$. Entre paréntesis aparece el coeficiente del efecto directo. $* p<.05, * * p<.01, * * * p<.001$.

\subsection{Conclusiones}

El estudio 1 arroja resultados que corroboran tres de las cuatro hipótesis planteadas. Se contrastó que la presencia de información sobre el origen nacional de los delincuentes indujo un efecto significativo en las actitudes y emociones negativas sentidas hacia los inmigrantes de manera específica cuando la implicación con el tema de la noticia era baja. Igualmente se observó que el proceso o mecanismo de influencia de la mención de información sobre el origen de los delincuentes en la activación de creencias estereotípicas estaba mediado por la actitud hacia la inmigración, pero únicamente entre los participantes de baja implicación. Ahora bien, esto no significa que las personas de alta implicación no sean susceptibles a este tipo de información periférica contenida en una noticia. Tal como se ha hipotetizado, en este caso el proceso de influencia es muy probable que esté relacionado con la mediación cognitiva, es decir, por la activación de corrientes de pensamiento. Esta hipótesis fue contrastada en el segundo estudio.

\section{Estudio 2}

El estudio 1 presentó una limitación importante: no se observaron diferencias en el interés ni en el impacto emocional de la noticia entre las personas expuestas a una noticia que aludía a un suceso ocurrido en su propia ciudad (Valladolid, alta implicación) en comparación las personas que leyeron una noticia que narraba el mismo suceso pero localizado en otra ciudad menos próxima (Barcelona, 
baja implicación). Una segunda limitación es no haber recogido información sobre las reacciones ante la noticia (respuestas cognitivas), ya que sólo se emplearon medidas de auto-informe. Por todo ello, se diseñó un segundo experimento con el fin de hacer frente a los problemas que se acaban de mencionar. El objetivo principal de este segundo estudio fue contrastar un modelo mediacional que incluyera como variables mediadoras las respuestas cognitivas suscitadas por la lectura de la noticia.

\subsection{Método}

El estudio 2 consistió en una investigación experimental, en la que participaron 276 estudiantes de la Universidad de Salamanca, siendo el 56.2\% mujeres, y con una media de edad de 19 años (DT = 2.49; de 17 a 46 años). Al igual que en el estudio 1 se utilizó un diseño 2 x 2, en función de dos manipulaciones experimentales realizadas en una noticia (group cue e implicación). Se utilizó la misma noticia empleada en el estudio 1 (únicamente el $4.7 \%$ de los participantes declaró que ya había leído con anterioridad la noticia), con las oportunas modificaciones, ya que para la manipulación de la implicación con el tema de la noticia se crearon dos condiciones: baja (el suceso delictivo sucede en Oviedo) o alta (el suceso delictivo sucede en Salamanca, la ciudad de los participantes en el estudio). Las variables dependientes fueron evaluadas mediante un cuestionario split-ballot que fue aplicado en varias aulas de la Universidad de Salamanca. En cada aula, los sujetos fueron aleatorizados a las cuatro condiciones experimentales. Una vez leída la noticia experimental, los participantes debían cumplimentar las preguntas del cuestionario relacionadas las variables dependientes, de chequeo de la manipulación y de control:

Respuestas cognitivas. Se evaluaron mediante la "tarea de listado de pensamientos" (Igartua y Cheng, 2009; Valkenburg, Semetko y de Vreese, 1999). A los sujetos se les pidió: "escribe todos los pensamientos, ideas o reflexiones que te haya suscitado la lectura de la noticia, es decir, aquellas impresiones que te surgieron durante la lectura de la noticia". Cuatro codificadores ${ }^{7}$ [7] con formación en análisis de contenido efectuaron el proceso de análisis. En primer lugar, se contabilizó el número total de respuestas cognitivas y el número total de palabras escritas por cada encuestado. A continuación, tomando como unidad de análisis cada una de las ideas escritas, se evaluaron los siguientes criterios: a) ¿se hace alusión al origen nacional de los atracadores o delincuentes, con expresiones como extranjero o inmigrante (o similares), o se hace un comentario sobre la inmigración o los extranjeros en general? $(1=$ sí, $0=$ no); b) ¿se manifiesta empatía, simpatía, lástima o identificación con las víctimas del asalto? $(1=$ sí, $0=$ no); c) ¿se manifiesta una reacción de indignación, desprecio, ira, rabia o repulsa por la situación relatada en la noticia, o hacia los atracadores? $(1=$ sí, $0=$ no); d) ¿se demandan medidas punitivas (como penas de cárcel más severas) o medidas policiales (mayor vigilancia o efectividad policial) para hacer frente al tipo de sucesos narrados en la noticia? $(1=$ sí, $0=$ no); e) ¿se alude a la falta de seguridad, o a la sensación de inseguridad y/o miedo en la sociedad? $(1=$ sí, $0=$ no); f $)$ ¿se utilizan insultos o expresiones negativas como el "carácter inhumano", la "frialdad", "brutalidad", "dureza", "cobardía", "maldad", "crueldad", "falta de escrúpulos" o similares, que aluden al carácter o personalidad de los asaltantes o delincuentes protagonistas de la noticia? $(1=$ sí, $0=$ no); g) ¿se señala una relación directa entre la inmigración y el delito y/o con la inseguridad ciudadana, como por ejemplo, cuando se dice que los inmigrantes son delincuentes o que con el aumento de la inmigración hay más delitos y/o inseguridad? $(1=$ sí, $0=$ no). Para evaluar la fiabilidad del proceso de codificación, se eligieron al azar 46 cuestionarios (el 16.66\% de la muestra, 137 respuestas cognitivas) y dos analistas codificaron de manera independiente los criterios mencionados. La fiabilidad se calculó mediante el coeficiente Alpha de Krippendorff (utilizando la macro KALPHA para SPSS; Hayes y Krippendorff, 2007), arrojando los siguientes valores: número de respuestas cognitivas escritas $\left(\alpha_{\mathrm{k}}=.95\right)$, número total de palabras escritas $\left(\alpha_{k}=.99\right)$, alusión al origen nacional de los delincuentes $\left(\alpha_{k}=1.0\right)$, alusión a 
expresiones de empatía $\left(\alpha_{k}=.82\right)$, alusión a expresiones de repulsa $\left(\alpha_{k}=.65\right)$, alusión a demanda de medidas punitivas o policiales $\left(\alpha_{k}=.83\right)$, alusión a la falta de seguridad $\left(\alpha_{k}=.58\right)$, referencias al carácter o personalidad de los delincuentes $\left(\alpha_{k}=.54\right)$ y alusiones a la existencia de una relación entre inmigración e inseguridad ciudadana $\left(\alpha_{\mathrm{k}}=.82\right)$. La media de respuestas cognitivas escritas fue de 3.41 ideas por persona $(D T=1.46)$ y la media de palabras escritas fue de 41.64 palabras por persona $(D T=27.30)$. Con la información procedente de la codificación de las respuestas cognitivas se creó un fichero de datos agregados (tomando como variable de agregación el número de cuestionario), de modo que en fichero final de datos (que contenía también la información sobre el resto de las variables) se indicaba el número y porcentaje de respuestas cognitivas que se centraban en cada uno de los aspectos señalados para cada participante.

Emociones sentidas durante la lectura de la noticia. Los participantes debían indicar en qué medida (desde 1 "nada" hasta 5 "mucho") la lectura de la noticia les había hecho sentir diez emociones diferentes (véase estudio 1).

Evaluación de la noticia. Se utilizó un diferencial semántico compuesto por 9 escalas bipolares (de 7 puntos) en cuyos extremos figuraban adjetivos antónimos como "clara-confusa", "de lectura fácil-de lectura difícil", etc. (véase estudio 1).

Interés suscitado por la noticia. Se preguntó a los participantes "valora el grado de interés personal que te ha suscitado la noticia" (desde 0 "ningún interés" hasta 10 "mucho interés").

Creencias sobre las consecuencias de la inmigración. Se construyó una escala con 8 afirmaciones sobre las consecuencias de la inmigración en España (véase ítems en estudio 1). Un análisis factorial de componentes principales (rotación varimax) extrajo dos factores (61.81\% de la varianza). El primer factor aludía a la creencia "la inmigración supone una contribución económica necesaria para el país" $(\alpha=.73)$; el segundo se refería a la creencia "la inmigración favorece el incremento de la delincuencia y la inseguridad ciudadana" $(\alpha=86)$.

Autoposicionamiento político. Los participantes debían contestar a la siguiente pregunta: "cuando se habla de política se utilizan normalmente las expresiones "izquierda" y "derecha". Teniendo en cuenta la siguiente escala, ¿qué número representaría mejor tu posición política?" (desde 1 "izquierda" hasta 10 "derecha"; $M=5.23, D T=2.38$ ).

Consumo de medios. Se preguntó: "en términos generales, ¿en un día normal de la semana cuántas horas ves televisión? ¿Escuchas radio? ¿Lees la prensa de información general? ¿Navegas por Internet?". Se creó un indicador de "consumo global de medios de comunicación" a partir de la suma simple de las cuatro variables consideradas $(M=396.96$ minutos al día, $D T=280.01)$.

Seguimiento de noticias. Se preguntaba: “¿en qué medida (desde 1 "nada” hasta 5 "mucho”) sueles seguir las noticias a través de los diferentes medios que se citan a continuación?" (radio, televisión, prensa escrita e Internet). Se creó un indicador de "consumo de noticias en los medios de comunicación" a partir de la suma simple de las cuatro variables consideradas $(M=12.44, D T=2.40)$.

Interés por el tema de la inmigración. Se preguntaba: ¿cuánto interés te suscita el tema de la inmigración? (desde 1 "nada" hasta 5 "mucho"; $M=3.60, D T=0.75$ ).

Participación en conversaciones sobre el tema de la inmigración. A los participantes se les planteó la siguiente pregunta: ¿Alguna vez has participado activamente, dando tu opinión, en conversaciones o discusiones sobre el tema de la inmigración? (desde 1 "nunca" hasta 5 "muchas veces"; $M=2.94$, $D T=1.14)$.

Nivel de contacto con inmigrantes. Se preguntaba al sujeto si mantenía, o había mantenido en el pasado $(1=$ sí, $0=$ no), algún tipo de relación familiar, de amistad, en el trabajo, de estudios o de 
vecindad con inmigrantes. Se creó un índice de contacto personal con inmigrantes a partir de la suma simple de las cinco variables dicotómicas consideradas $(M=2.32, D T=1.25)$.

Experiencia personal con el delito. Se preguntaba a los participantes si, a lo largo de su vida: “¿has sido víctima de algún robo en el que no hubo agresión física?" ( $1=$ sí, $0=$ no; $36.2 \%$ de respuestas afirmativas) y “¿has sido víctima de algún robo o atraco con agresión física?” ( $1=\mathrm{sí}, 0=$ no; $4.0 \%$ de respuestas afirmativas). Se creó un índice de experiencia personal con el delito sumando los valores de dichas variables $(M=0.39, D T=0.52)$.

\subsection{Resultados}

En primer lugar, se realizó un análisis de varianza de un factor (ANOVA) en el que se incluyó como variable independiente la condición experimental (compuesta por cuatro grupos) y como variables dependientes los nueve indicadores de evaluación de la noticia. Los resultados mostraron que no existían diferencias estadísticamente significativas entre las cuatro versiones de la noticia experimental en ninguna de las variables de evaluación de la noticia (en todos los análisis $p>.296$ ). Estos resultados indican que las cuatro noticias experimentales, a pesar de contener dos manipulaciones, no generaron diferencias en cuanto a su valoración general y por tanto se comportaron como estímulos homogéneos. Tampoco se observaron diferencias significativas entre los cuatro grupos experimentales en función del sexo, la edad, el auto-posicionamiento político, el interés por el tema de la inmigración, la participación en conversaciones sobre el tema de la inmigración, el nivel de contacto con inmigrantes, la experiencia con el delito, el consumo de medios y el seguimiento de noticias (en todos los análisis $p>$.330). Por tanto, se puede concluir que el procedimiento de aleatorización de los participantes a las condiciones fue exitoso y condujo a la formación de cuatro grupos homogéneos en variables relevantes.

En el indicador de interés suscitado por la noticia, no se observaron diferencias estadísticamente significativas entre los participantes que leyeron la noticia emplazada en Salamanca $(M=6.73$, $D T=1.85$ ) y los que fueron expuestos a una noticia que aludía a los mismos hechos pero situados en Oviedo $(M=6.55, D T=1.90)(t(274)=-0.81, p=.419)$. En cuanto al impacto emocional de la noticia, sólo se observó una diferencia estadísticamente significativa con respecto a la emoción de sorpresa $(t(270)=-2.66, p<.008)$. Los participantes que leyeron una noticia que hacía referencia a un hecho delictivo en Salamanca $(M=2.66, D T=1.05)$ mostraron mayor sorpresa que los participantes expuestos a la misma noticia pero situada en Oviedo $(M=2.33, D T=0.98)$. A raíz de estos resultados se puede concluir que la manipulación de la implicación con la noticia no produjo el efecto esperado en el interés y en las emociones, lo que sugiere que el procedimiento empleado para manipular la implicación con la noticia no es totalmente adecuado.

Para contrastar la hipótesis 5 se efectuaron nueve análisis de covarianza (ANCOVA) para un diseño factorial 2 x 2 de medidas independientes, incluyéndose como covariables el sexo, la edad, el autoposicionamiento político, el interés por el tema de la inmigración, la participación en conversaciones sobre el tema de la inmigración, el nivel de contacto con inmigrantes, la experiencia con el delito, el consumo de medios y el seguimiento de noticias. Con la inclusión de covariables, se obtiene información sobre el impacto de las variables independientes a margen de la influencia de otras variables relevantes, con lo que se incrementa la potencia estadística de los análisis (Hayes, 2005). Las variables dependientes consideradas fueron los diferentes indicadores creados de respuestas cognitivas (RC; véase Método). 
Figura 4. Efecto de la información sobre el origen de los delincuentes y de la implicación con el tema de la noticia en el número total de RC escritas

\section{Número total de respuestas cognitivas}

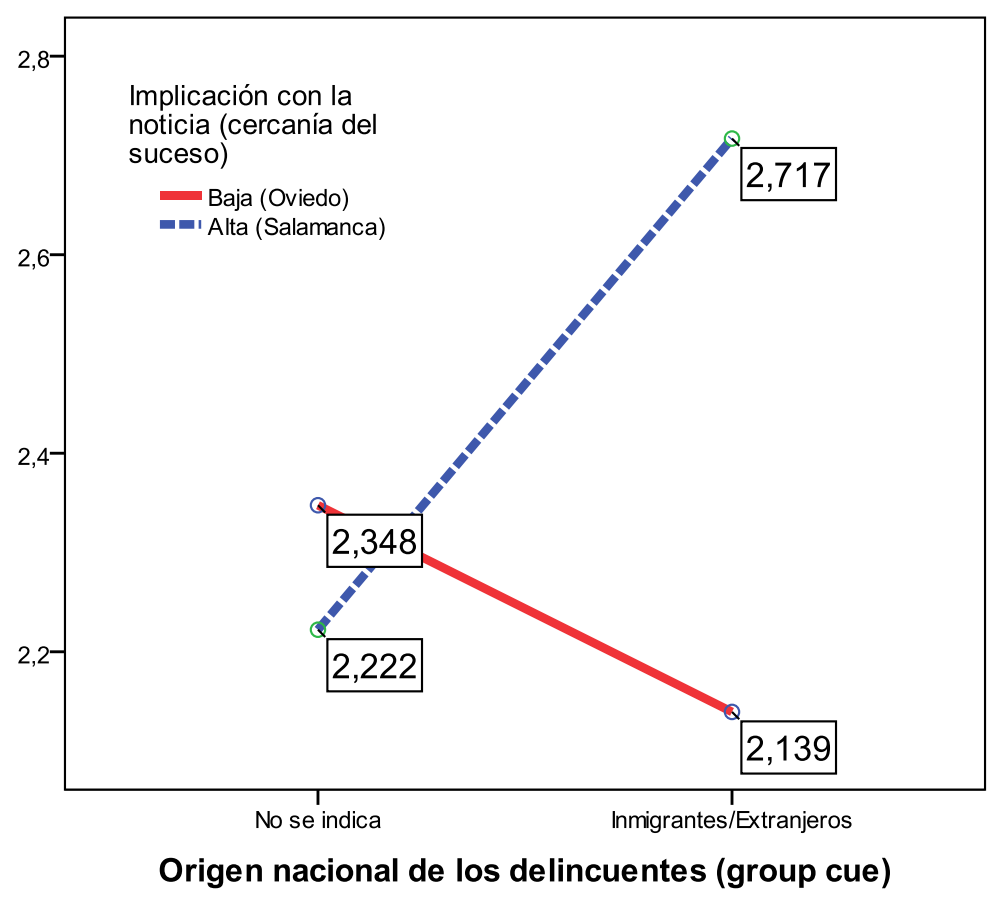

Figura 5. Efecto de la información sobre el origen de los delincuentes y de la implicación con el tema de la noticia en el número total de palabras escritas

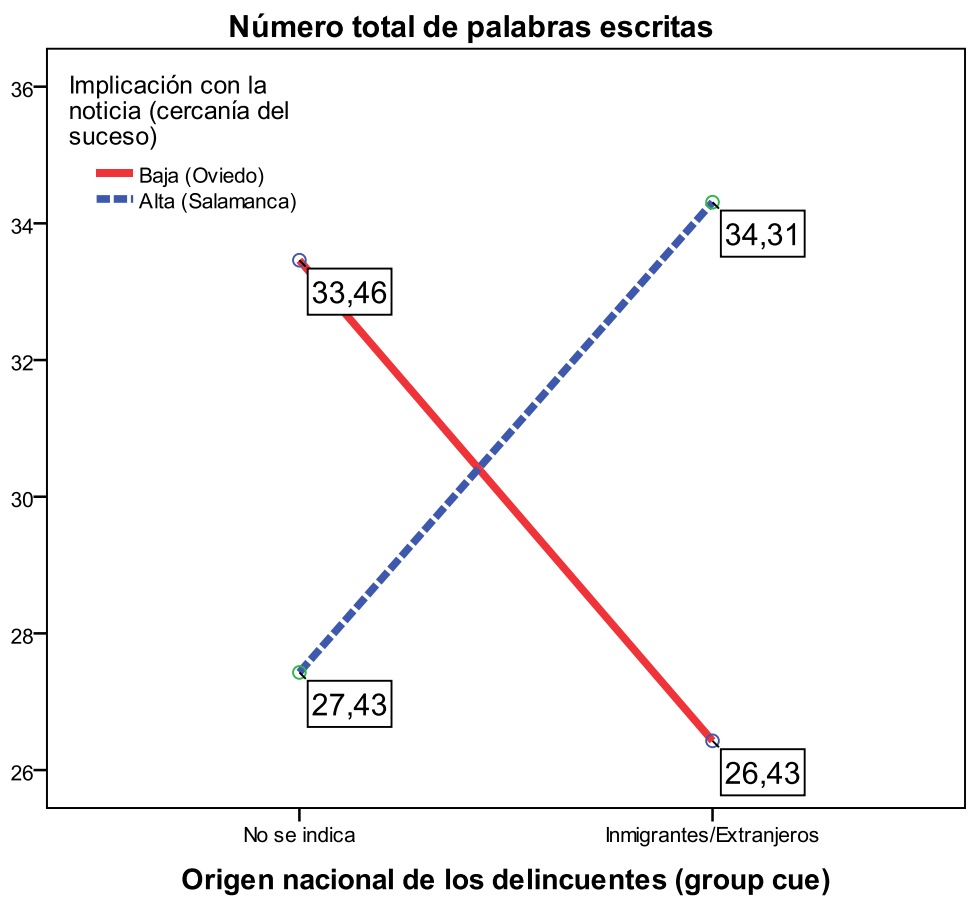


Se obtuvo un efecto de interacción tendencial en el "número total de $\mathrm{RC}$ " $(F(1,189)=2.94, p<.088$, $\left.\eta_{\mathrm{p}}{ }^{2}=.015\right)$. No se apreciaban diferencias entre los participantes de alta y baja implicación cuando no se indicaba el origen nacional de los delincuentes; en cambio, entre los estudiantes que leyeron una noticia que indicaba que los delincuentes eran inmigrantes se apreciaban claras diferencias en función de la implicación, escribiendo más respuestas cognitivas los participantes con alta implicación (véase Figura 4). Se obtuvo un resultado similar con respecto al "número total de palabras escritas" por los participantes (Figura 5). En este caso, el efecto de interacción tendencial $\left(F(1,189)=3.40, p<.067, \eta_{\mathrm{p}}^{2}=.018\right)$ ponía de manifiesto que los participantes con alta y baja implicación reaccionaban de manera muy diferente en función de que se incluyera o no información sobre el origen nacional de los delincuentes.

Figura 6. Efecto de la información sobre el origen de los delincuentes y de la implicación con el tema de la noticia en el porcentaje de RC que aluden al origen nacional de los delincuentes

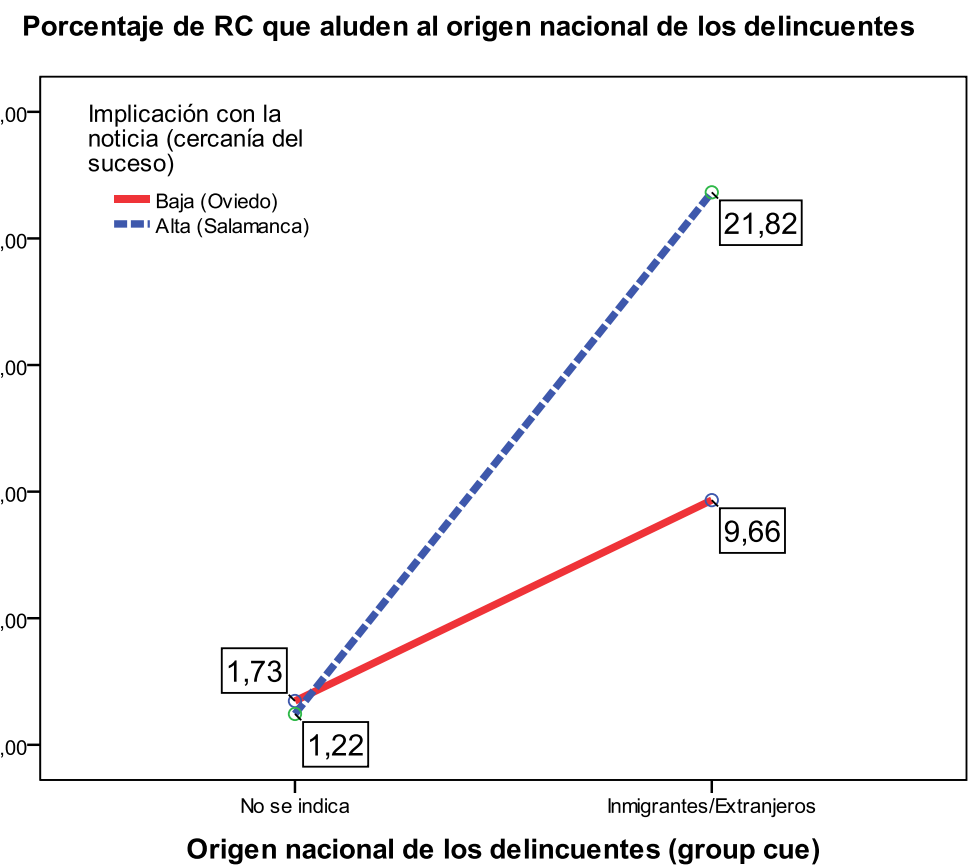

En la variable "porcentaje de RC que aluden al origen nacional de los delincuentes" se observó un efecto de interacción significativo entre ambas variables independientes $(F(1,160)=4.02, p<.042$, $\eta_{\mathrm{p}}{ }^{2}=.026$ ), apreciándose que eran las personas con alta implicación, frente a las de baja, las que se vieron más afectadas por la noticia que contenía información sobre el origen nacional de los delincuentes (Figura 6). Igualmente, se observó un efecto de interacción estadísticamente significativo en los comentarios escritos por los participantes que vinculaban la inmigración con el incremento de la inseguridad ciudadana $\left(F(1,160)=7.07, p<.009, \eta_{\mathrm{p}}{ }^{2}=.042\right)$ : ante una noticia que contenía información sobre el origen nacional de los delincuentes, fueron las personas con alta implicación las que en mayor medida escribieron respuestas cognitivas en las que asociaban inmigración e inseguridad ciudadana (Figura 7). 
Figura 7. Efecto de la información sobre el origen de los delincuentes y de la implicación con el tema de la noticia en el porcentaje de $\mathrm{RC}$ con alusiones a la existencia de una relación entre inmigración e inseguridad ciudadana

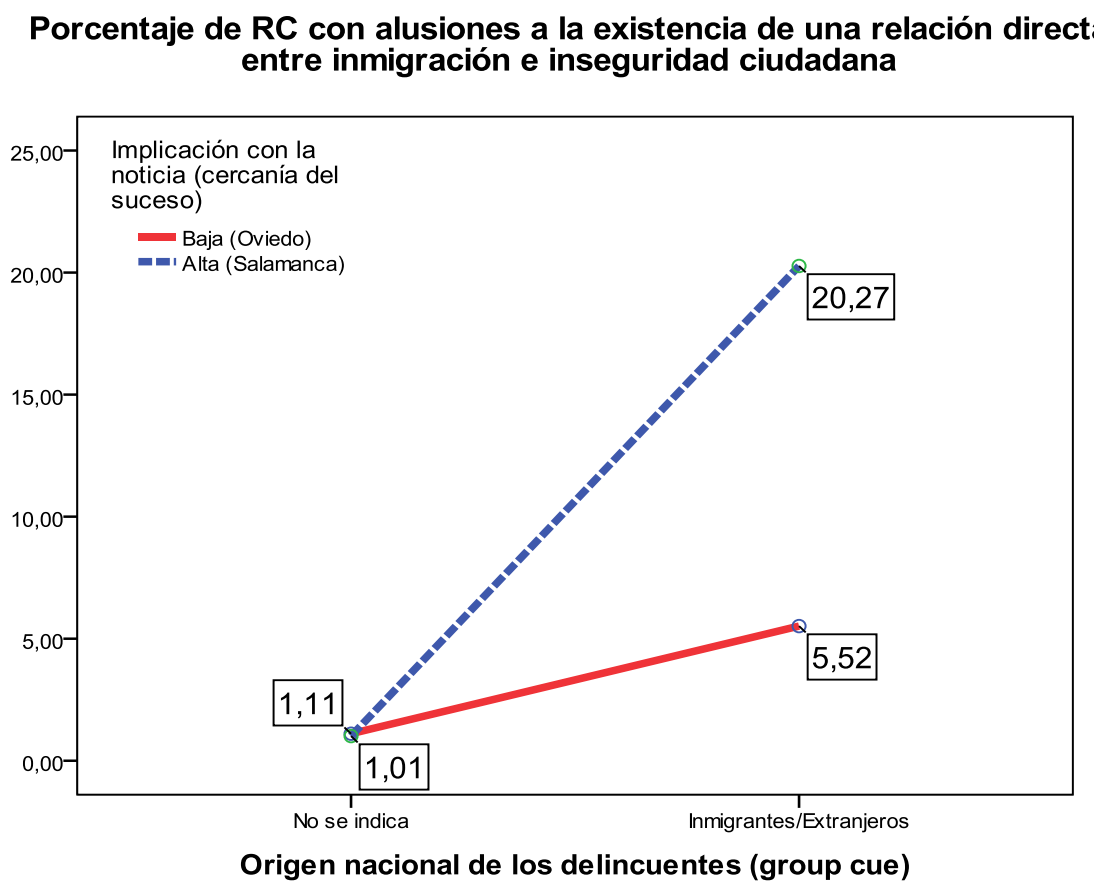

Para contrastar la hipótesis 6 se utilizó nuevamente la macro PROCESS (modelo 4) para SPSS elaborada por Hayes (2013), contrastándose un modelo mediacional con múltiples variables mediadoras (Preacher y Hayes, 2008), ya que se introdujeron como tales los siete indicadores de respuestas cognitivas obtenidos en la tarea del listado de pensamientos (todos excepto el número de RC y el número de palabras escritas), siendo la variable independiente la presencia o ausencia sobre de información sobre el origen nacional de los delincuentes (variable independiente, codificada como variable dummy, siendo $1=$ en la noticia se alude a que los delincuentes son inmigrantes, $0=$ no se menciona el origen nacional de los delincuentes), y la variable dependiente el grado de acuerdo con la creencia negativa y estereotípica "la inmigración favorece el incremento de la delincuencia y la inseguridad ciudadana". El modelo mediacional fue computado de manera separada para los participantes de alta y baja implicación (calculado utilizando 10.000 bootstrap samples), esperándose que las variables cognitivas solo tuvieran un efecto mediador significativo entre los participantes de alta implicación.

El análisis mediacional ejecutado entre los participantes de baja implicación reveló que ninguna de las siete variables relacionadas con el impacto cognitivo de la noticia actuaba como variable mediadora y, por tanto, no se observaron efectos indirectos estadísticamente significativos. En cambio, el análisis mediacional entre los participantes de alta implicación reveló un efecto indirecto estadísticamente significativo a través de la variable mediadora "RC sobre la existencia de una relación entre la inmigración con el incremento de la inseguridad ciudadana" $\left(B_{\text {efecto indirecto }}=.21\right.$, $S E=.11,95 \% C I[.02, .47]$; Figura 8). De este modo, mencionar en la noticia que los atracadores eran inmigrantes indujo un elevado número de respuestas cognitivas que aludían a la relación entre inmigración e inseguridad ciudadana $(B=.31, p<.001)$, lo que se asoció a su vez a un mayor grado de acuerdo con la creencia estereotípica "la inmigración favorece el incremento de la delincuencia y la inseguridad ciudadana" $(B=.69, p<.042)$. 
Figura 8. Análisis mediacional (alta implicación, $\mathrm{n}=116$, estudio 2): las respuestas cognitivas suscitadas por la lectura de la noticia como variables mediadoras en la relación entre la mención de la información sobre el origen nacional de los protagonistas de la noticia y las creencias estereotípicas negativas sobre la inmigración

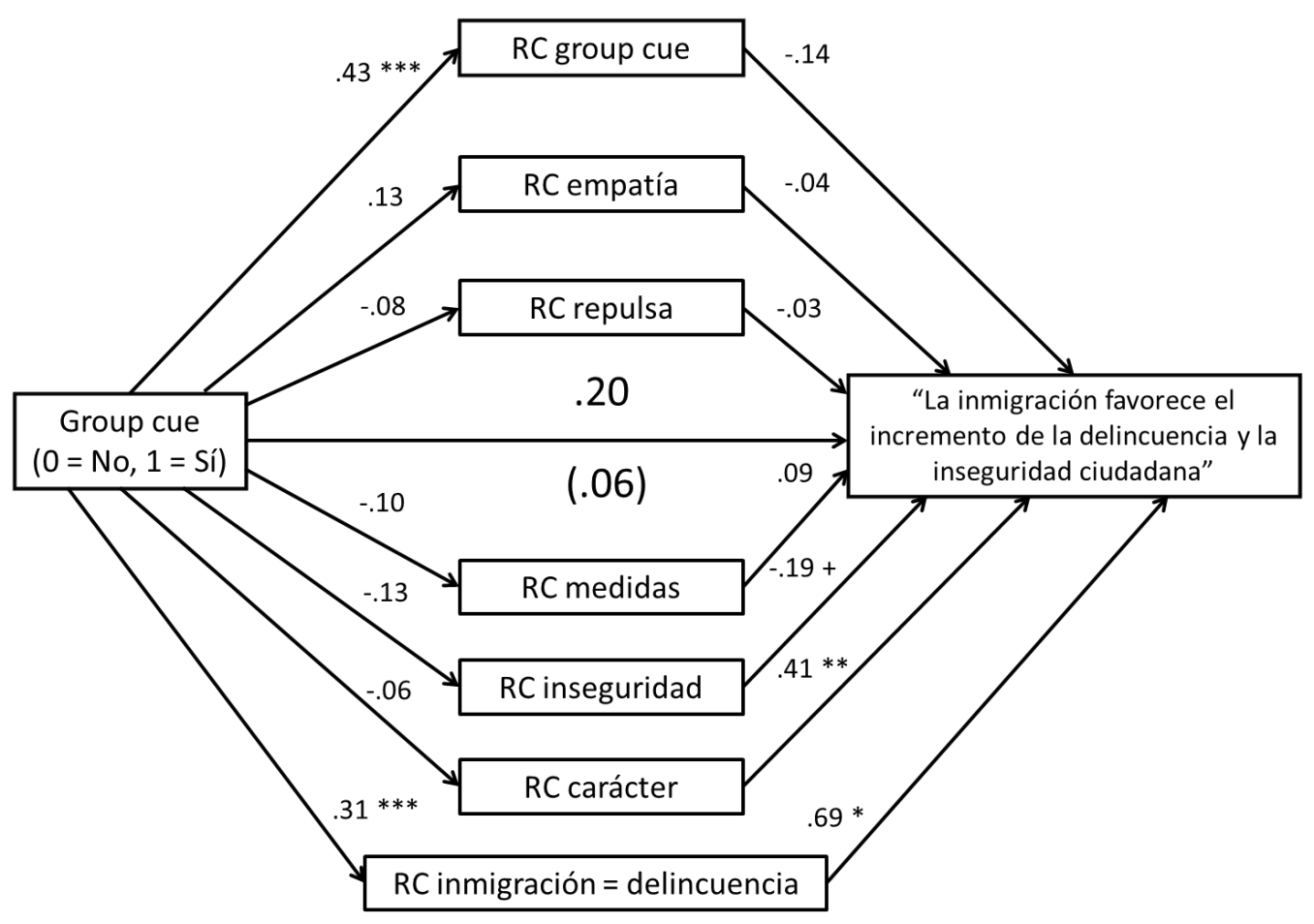

Group cue -> RC inmigración = delincuencia $->$ La inmigración favorece la delincuencia

$$
B_{\text {efecto indirecto }}=.21, S E=.11,95 \% \text { CI }[.02, .47]
$$

Nota. En la figura se muestran los coeficientes de regresión no estandarizados, $B$. Entre paréntesis aparece el coeficiente del efecto directo. $+p<.10, * p<.05, * * p<.01, * * * p<.001$

\subsection{Conclusiones}

Los resultados obtenidos en el estudio 2 suponen un apoyo sustancial a las hipótesis planteadas. De manera congruente con la hipótesis 5, la implicación con la noticia incrementó la producción cognitiva (el grado de reflexión y en número de palabras escritas) de los participantes especialmente cuando se indicaba que los delincuentes eran inmigrantes. Además, ante una noticia que contenía información sobre el origen nacional de los delincuentes, fueron las personas con alta implicación las que en mayor medida escribieron respuestas cognitivas en las que vinculaban inmigración e inseguridad ciudadana. Los resultados del modelo mediacional también son congruentes con la hipótesis 6 planteada. Así, se ha podido contrastar que las respuestas cognitivas (en particular, las emisión de respuestas cognitivas que aludían a una relación entre la inmigración y la inseguridad ciudadana) explicaban el efecto de la mención del origen nacional de los atracadores en la activación de los estereotipos sobre los inmigrantes pero únicamente cuando la implicación con el tema de la noticia era alta. 


\section{Conclusiones y discusión general}

Los dos estudios experimentales realizados tuvieron como objetivo principal comprobar los mecanismos o procesos que explican el impacto socio-cognitivo provocado por el encuadre dominante en el tratamiento informativo de la inmigración (que vincula la inmigración con la delincuencia y el incremento de la inseguridad ciudadana). Ambos estudios se diseñaron tomando como referencia las investigaciones sobre el efecto framing, el procesamiento cognitivo de las comunicaciones persuasivas y el papel de las claves (textuales o visuales) sobre el origen racial o grupal de los protagonistas de las noticias en la activación de los estereotipos (por ejemplo, Brader et al., 2008; Igartua y Cheng, 2009; Igartua, Moral y Fernández, 2011; Peffley, Shields y Williams, 2001).

Ante una noticia que narraba un acto delictivo de gran violencia, y en la que se habían manipulado la información sobre el origen de los delincuentes (group cue) y el lugar de los hechos (implicación), se contrastó que los participantes mostraban una actitud más negativa hacia la inmigración y experimentaron más emociones negativas hacia los inmigrantes cuando leyeron una noticia protagonizada por delincuentes etiquetados como inmigrantes y la implicación era baja, confirmándose las hipótesis 1 y 3. Sin embargo, este mismo efecto de interacción no tuvo lugar en relación con el impacto en las creencias estereotípicas negativas ("la inmigración favorece el incremento de la delincuencia y la inseguridad ciudadana"), por lo que la hipótesis 2 no recibió apoyo empírico. Ahora bien, sí se observó un efecto indirecto en dichas creencias, ya que los participantes con baja implicación mostraron un mayor grado de acuerdo con las creencias estereotípicas cuando en la noticia se hacía mención al origen inmigrante de los atracadores y dicho efecto estaba mediado por la actitud hacia la inmigración. Por tanto, informar sobre el origen de los delincuentes ejercía un efecto indirecto en la activación de los estereotipos pero únicamente cuando se había inducido baja implicación en los participantes, lo que corrobora la hipótesis 4.

En el estudio 2 se recogieron y analizaron las respuestas cognitivas suscitadas por la lectura de la noticia mediante una tarea de listado de pensamientos, observándose que los participantes con alta y baja implicación reaccionaron de manera muy diferente en función de que se incluyera o no información sobre el origen nacional de los delincuentes. Así, la implicación con el tema de la noticia incrementó la producción cognitiva de los participantes pero únicamente cuando se indicaba que los delincuentes eran inmigrantes. Además, se produjo un efecto de canalización cognitiva y respuestas cognitivas más críticas hacia la inmigración entre los participantes a los que se había inducido una alta implicación con la noticia protagonizada por delincuentes definidos como inmigrantes o extranjeros, lo que confirma la hipótesis 5. También se corroboró la hipótesis 6 , ya que las respuestas cognitivas críticas o negativas que aludían a la existencia de una relación entre la inmigración y la inseguridad ciudadana explicaban el efecto de la mención del origen nacional de los atracadores en la activación de los estereotipos sobre los inmigrantes pero únicamente cuando la implicación con el tema de la noticia era alta.

Los resultados obtenidos son convergentes con la propuesta teórica avanzada y con un modelo dual sobre los efectos de los encuadres noticiosos. Se constata que las claves periféricas presentes en un información periodística (como mencionar en una noticia sobre un hecho delictivo que los delincuentes eran inmigrantes o extranjeros, group cue) produce efectos socio-cognitivos significativos en las respuestas cognitivas, en las actitudes y creencias y en las emociones sentidas. Sin embargo, el mecanismo que explica dichos efectos es diferente en función del nivel de implicación de las personas con el acontecimiento narrado. Cuando el suceso acontece fuera del contexto cercano del individuo (baja implicación) la influencia de las claves periféricas se debe a que dicho atributo de la noticia activa una especie de atajo mental, un juicio rápido hacia el objeto que ha 
sido resaltado en la información (en este caso, que los delincuentes son inmigrantes o extranjeros) produciéndose un proceso de modelado actitudinal. Es decir, la clave periférica activa una determinada actitud y ésta a su vez influye en la activación, formación o refuerzo de una determinada creencia u opinión. Este mecanismo no se pondría en marcha entre las personas con alta implicación con el tema, lo cual no significa que no se vean afectadas por las claves periféricas. Así, cuando las personas leen una información sobre un acontecimiento que ha tenido lugar en su propio contexto cercano (alta implicación), las claves periféricas influyen en los procesos de reflexión activándose un tipo de procesamiento cognitivo sesgado, que conduce a una proceso de canalización cognitiva: las repuestas cognitivas escritas por los individuos son del mismo tono que la información que aparece en la noticia, lo que determinará a su vez el impacto en las opiniones o creencias.

Esta propuesta teórica sobre los mecanismos de influencia de los encuadres noticiosos se ha contrastado en un contexto específico: el tratamiento de la inmigración en los medios. Con objeto de verificar su validez en un contexto más general, la investigación futura debería centrarse en otros temas. Además, también se debería tener en cuenta en futuras investigaciones el efecto de otras claves periféricas en la construcción de los encuadres noticiosos, más allá de la alusión al origen de los protagonistas de la información. Por otro lado, deberían desarrollarse investigaciones que analicen los efectos a largo plazo en condiciones de alta y baja implicación ya que, de acuerdo con el ELM, las actitudes formadas o cambiadas a través de la ruta central muestran una mayor persistencia temporal, un mayor poder predictivo de la conducta y mayor resistencia a la contra-argumentación que si éstas han sido formadas o cambiadas a través de la ruta periférica (Petty y Cacioppo, 1986). Aunque estas predicciones han sido contrastadas en el campo de la persuasión no existe evidencia empírica hasta la fecha sobre si estos procesos también operan con respecto a los efectos de los encuadres noticiosos.

A pesar que de los dos estudios experimentales tuvieron niveles satisfactorios de calidad (aleatorización de los participantes a los tratamientos, estímulos homogéneos, adecuada consistencia interna de las escalas), cabe señalar una serie de limitaciones. En primer lugar, el procedimiento para manipular la implicación no funcionó adecuadamente. De todos modos, la clave periférica group cue sí ejerció un efecto indirecto significativo en la activación de las creencias estereotípicas y negativas sobre la inmigración a través de la mediación de las actitudes (baja implicación) y de las respuestas cognitivas (alta implicación). Una segunda limitación es que en ambas investigaciones participaron estudiantes universitarios, es decir, un grupo muy homogéneo en cuanto a sus actitudes hacia los inmigrantes. Sería recomendable replicar este tipo de investigaciones con población general. En tercer lugar, otra posible limitación se refiere al tipo de variables dependientes utilizadas (escalas auto-aplicadas y tarea de listado de pensamientos). Quizá se habrían detectado efectos relevantes más fácilmente utilizando medidas implícitas de las actitudes (Briñol, Petty y McCaslin, 2009; Dovidio, Kawakami, Smoak y Gaertner, 2009).

-Este artículo es producto del proyecto de investigación titulado "Análisis y efectos sociocognitivos de los encuadres noticiosos de la inmigración en la prensa regional de Castilla y León", con referencia $n^{\circ}$ SA040A06, financiado por el Programa de Apoyo a Proyectos de Investigación de la Consejería de Educación de la Junta de Castilla y León.

\section{Referencias bibliográficas}

Baron, R. M. y Kenny, D. A. (1986): The moderator--mediator variable distinction in social psychological research: Conceptual, strategic and statistical considerations. Journal of Personality and Social Psychology, 51(6), 1173-1182. 
Brader, T., Valentino, N. A. y Suhay, E. (2008): What triggers public opposition to immigration? Anxiety, group cues, and immigration threat.American Journal of Political Science, 52(4), 959-978.

Briñol, P., Petty, R. E. y McCaslin, M. J. (2009): Changing attitudes on implicit versus explicit measures: what is the difference? En R. E. Petty, R. H. Fazio, y P. Briñol (Eds.), Attitudes: Insights from the new implicit measures (pp. 429-458). Nueva York, NY: PsychologyPress.

Cea D’Ancona, M. A. (2004): La activación de la xenofobia en España. Madrid: CIS-Siglo XXI. deVreese, C. H. (2003): FramingEurope. Television news and European integration. Amsterdam: Aksant.

Dovidio, J. F., Kawakami, K., Smoak, N. y Gaertner, S. L. (2009): The nature of contemporary racial prejudice: insights from implicit and explicit measures of attitudes. En R. E. Petty, R. H. Fazio, y P. Briñol (Eds.), Attitudes: Insights from the new implicit measures (pp. 429-458): Nueva York, NY: Psychology Press.

Entman, R. (1993). Framing: toward a clarification of a fractured paradigm. Journal of Communication, 43(4), 51-58.

Gilliam, F. D., Iyengar, S., Simon, A. y Wright, O. (1996): Crime in black and white.The violent, scary world of local news.Press/Politics, 1(3), 6-23.

Gross, K. y Brewer, P. R. (2007): Sore losers: news frames, policy debates, and emotions. Harvard International Journal of Press/Politics, 12(1), 122-133.

Gross, K. y D’Ambrosio, L. (2004): Framing emotional response. Political Psychology, 25(1), 1-29.

Hayes, A. F. (2005): Statistical methods for communication science. Mahwah, NJ: Lawrence Erlbaum Associates.

Hayes, A. F. (2009): Beyond Baron and Kenny: statistical mediation analysis in the new millennium. Communication Monographs, 76(4), 408-420.

Hayes, A. F. (2013): Introduction to mediation, moderation and conditional process analysis.A regression-based approach. Nueva York, NY: The Guilford Press.

Hayes, A. F. y Krippendorff, K. (2007): Answering the call for a standard reliability measure for coding data.Communication Methods and Measures, 1(1), 77-89.

Igartua, J. J. y Cheng, L. (2009): Moderating effect of group cue while processing news on immigration. Is framing effect a heuristic process? Journal of Communication, 59(4), 726-749.

Igartua, J. J., Cheng, L. y Lopes, O. (2003): To think or not to think: two pathways towards persuasion by short films on Aids prevention. Journal of Health Communication, 8(6), 513-528.

Igartua, J. J., Moral, F. y Fernández, I. (2011): Cognitive, attitudinal and emotional effects of the news frame and group cues on processing news about immigration. Journal of Media Psychology, 23(4), 174-185.

Iyengar, S. (1991): Is anyone responsible? How television frames political issues. Chicago: The University of Chicago Press.

Johnson, B. T. y Eagly, A. H. (1990): Involvement and persuasion: types, traditions and the evidence. Psychological Bulletin, 107(3), 375-384.

Machill, M., Köhler, S. y Waldhauser, M. (2007):The use of narrative structures in television news.An experiment in innovative forms of journalistic presentation.European Journal of Communication, 22(2), 185-205. 
Matthes, J. (2007): Beyond accessibility? Toward an on-line and memory-based model of framing effects.Communications: The European Journal of Communication Research,32(1), 51-78.

McCombs, M. E., Lopez-Escobar, E. y Llamas, J. P. (2000): Setting the agenda of attributes in the 1996 Spanish general election.Journal of Communication, 50(2), 77-92.

McCombs, M. E. y Reynolds, A. (2002): News influence on our pictures of the world.En J. Bryant y D. Zillmann (Eds.), Media effects.Advances in theory and research (pp. 1-18):Mahwah, NJ: Lawrence ErlbaumAssociates.

Navas, M., García, M. C., Rojas, A. J., Pumares, P. y Cuadrado, I. (2006). Actitudes de aculturación y prejuicio: la perspectiva de autóctonos e inmigrantes. Psicothema, 18(2), 187-193.

Peffley, M., Shields, T. y Williams, B. (2001): The intersection of race and crime in television news stories: an experimental study. Political Communication, 13(3), 309-327.

Perse, E. M. (2001): Media effects and society. Mahwah, NJ: Lawrence Erlbaum Associates.

Petty, R. E. y Cacioppo, J. T. (1986): Communication and persuasion. Central and peripherical routes to attitude change. Nueva York: Springer-Verlag.

Petty, R. E., Priester, J. R. y Wegener, D. T. (1994): Cognitive processes in attitude change. En R. S. Wyer y T. K. Srull (Eds.), Handbook of social cognition. Volume 2: applications (pp. 69-141): Hillsdale, NJ: Lawrence Erlbaum Associates.

Preacher, K. J. y Hayes, A. F. (2008): Asymptotic and resampling strategies for assessing and comparing indirect effects in multiple mediator models. Behavior Research Methods, 40(3), 879891.

Price, V. y Tewksbury, D. (1997): News values and public opinion: A theoretical account of media priming and framing. En G. Barnett y F. J. Foster (Eds.), Progress in communication sciences (pp. 173-212). Greenwich, CT: Ablex.

Price, V., Tewksbury, D. y Powers, E. (1997): Switching trains of thought. The impact of news frames on reader's cognitive responses. Communication Research, 24(5), 481-506.

Scheufele, D. (1999): Framing as a theory of media effects.Journal of Communication, 49(1), 103122.

Scheufele, D. (2000): Agenda-setting, priming and framing revisited: another look at cognitive effects of political communication. Mass Communication and Society, 3(2-3), 297-316.

Sendín, J. C. e Izquierdo, P. (2008):Guía práctica para los profesionales de los medios de comunicación: tratamiento mediático de la inmigración. Madrid: OBERAXE, Ministerio de Trabajo y Asuntos Sociales.

Tankard, J. W. (2001): The empirical approach to the study of media framing. En S. D. Reese, O. H. Gandy y A. E. Grant (Eds.), Framing public life (pp. 95-106). Mahwah, NJ: Lawrence Erlbaum Associates.

Valkenburg, P. M., Semetko, H. A. y De Vreese, C. H. (1999): The effects of news frames on readers' thoughts and recall.Communication Research, 26(5), 550-569.

Von Sikorski, C. y Schierl, T. (2012): Effects of news frames on recipients' information processing in disability sports communications.Journal of Media Psychology, 24(3), 113-123. 


\section{Notas}

[1] Véase: "Recomendaciones del CAC sobre el tratamiento informativo de la inmigración" http://www.cac.cat/pfw_files/cma/recerca/quaderns_cac/Q12recomanacions_ES.pdf

[2] La investigación sobre efectos mediáticos está cada vez más enfocada hacia el análisis de los procesos o mecanismos mediadores. Los trabajos de Baron y Kenny (1986) y los más recientes de Hayes (2009) han servido para dotar a los investigadores de herramientas metodológicas que permiten el contraste de los procesos mediadores y, por tanto, obtener un conocimiento más profundo y avanzado sobre la evidencia causal acerca de los efectos mediáticos (Hayes, 2013). La investigación sobre el efecto framing se ha visto influida por esta corriente de análisis mediacionales. En este contexto, no solo se trata de determinar si un determinado enfoque o encuadre noticioso influye en los procesos cognitivos, actitudinales y emocionales sino de explicar y explicitar el mecanismo responsable, es decir, por qué se produce una determinada influencia en función de cómo se construya una historia informativa.

[3] Para realizar el trabajo de campo se contó con la colaboración del profesor José Antonio Otero, docente de dicha universidad y miembro del Observatorio de los Contenidos Audiovisuales de la Universidad de Salamanca.

[4] En la siguiente dirección se puede acceder a la versión online de la noticia: http://elpais.com/diario/2006/06/18/espana/1150581613_850215.html

[5] Se creó una nueva variable condición (compuesta por cuatro grupos) a partir de combinar los niveles de las dos variables independientes.

[6] Según el método bootstrapping, un efecto indirecto es estadísticamente significativo si el intervalo de confianza establecido (CI al 95\%) no incluye el valor 0 . Si el valor 0 está incluido en dicho intervalo de confianza no se puede rechazar la hipótesis nula que plantea que el efecto indirecto es igual a 0, es decir, que no existe asociación entre las variables implicadas (Hayes, 2013).

[7] Los codificadores fueron: Elena Palacios, Salvador Alvidrez, Socorro Palitó y Tania Acosta.

\section{CÓMO CITAR ESTE ARTÍCULO EN BIBLIOGRAFÍAS - HOW TO CITE THIS ARTICLE IN BIBLIOGRAPHIES / REFERENCES:}

JJ Igartua (2013): "Impacto actitudinal y canalización cognitiva de estereotipos sobre la inmigración a través de las noticias", en Revista Latina de Comunicación Social, 68, 599 a 621.

Artículo recibido el 22 de julio de 2013. Sometido a pre-revisión el 24 de julio. Enviado a revisores el 26 de julio. Aceptado el 19 de septiembre de 2013. Galeradas telemáticas a disposición del autor el 19 de septiembre de 2013. Visto bueno del autor: 3 de octubre de 2013. Publicado el 4 de octubre de 2013. 